This manuscript is a preprint and has been submitted for publication in One Earth. Please note that the manuscript has not yet undergone peer-review and is yet to be accepted for publication. Subsequent versions of this manuscript may have slightly different content. If accepted, the final version of this manuscript will be available via the 'Peerreviewed Publication DOI'link on the right-hand side of this webpage. Please feel free to contact the authors with any comments, we welcome feedback. 


\title{
Heat Does Not Physically Flow in The Ways Assumed by Greenhouse-Warming Theory
}

\author{
Author \\ Peter L. Ward

\section{Correspondence} \\ peward@,wyoming.com
}

\section{Graphical Abstract}

Planck's empirical law shows that temperature is the result of a broad spectrum of frequencies of oscillation of all the bonds holding solid matter together

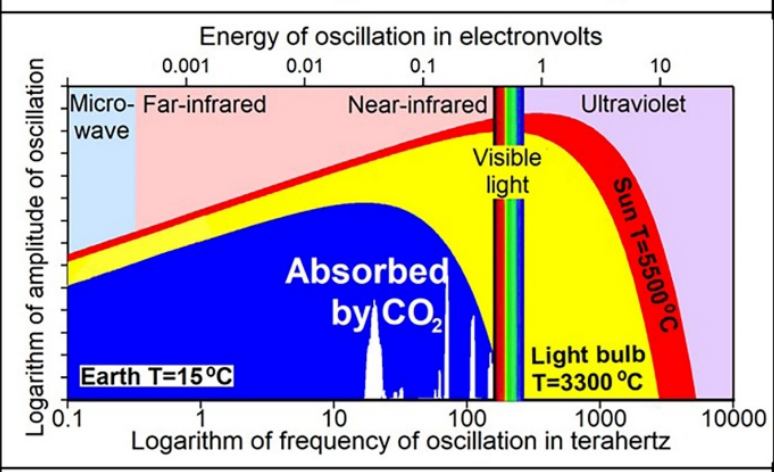

$\mathrm{CO}_{2}$ absorbs less than $16 \%$ of these frequencies as shown in white

\section{In Brief}

The way physicists have thought about heat for two centuries fails catastrophically for the large temperature difference between $\operatorname{Sun}\left(5500^{\circ} \mathrm{C}\right)$ and Earth $\left(15^{\circ} \mathrm{C}\right)$. It does not consider major increases in radiant thermal energy as a function of frequency of oscillation of all the bonds holding solid matter together. It grossly overestimates the thermal effects of infrared radiation absorbed by greenhouse gases. Greenhouse-warming theory cannot physically explain observed global warming. Ozone depletion theory provides a much clearer and more detailed explanation.

\section{Highlights}

- Temperature is the result of oscillation of all bonds holding solid matter together

- Amplitudes of oscillation increase with temperature as calculated by Planck's law

- Heat flows at each frequency by resonance of pairs of oscillators on body surfaces

- Greenhouse-warming theory cannot physically explain observed global warming 


\title{
Heat Does Not Physically Flow in The Ways Assumed by Greenhouse-Warming Theory
}

\author{
Peter L. Ward ${ }^{1,2, *}$ \\ ${ }^{1}$ U.S. Geological Survey retired \\ ${ }^{2}$ Science Is Never Settled, Inc., P.O. Box 4875, Jackson, WY 83001, USA \\ * Correspondence: peward@wyoming.com
}

Science for Society: Direct and unambiguous observations of radiation emitted by bodies of solid matter because of their temperature show that visible light and more generally electromagnetic radiation consist of a broad spectrum of frequencies of oscillation of all the bonds holding solid matter together. The higher the temperature, the higher the amplitude of oscillation at each and every frequency of oscillation. These amplitudes "flow" simultaneously by resonance between oscillators oscillating at the same frequency on the emitting and absorbing surfaces.

This new way of looking at radiation shows that heat simply does not flow in the ways assumed by greenhouse-warming theory. Greenhouse-warming theory is not only mistaken, it is not even physically possible. We may burn fossil fuels safely provided we minimize pollution. Observed global warming can be explained in detail by ozone-depletion theory, which predicts gradual global cooling throughout the rest of this century as the ozone layer recovers.

Summary: Heat is currently defined as an amount of thermal energy flowing each second per unit area. Temperature is assumed to result from the net amount of heat flowing - the sum of all radiative forcings. Yet direct and unambiguous observations of Nature show that macroscopic temperature of solid matter results from a very broad spectrum of sub-microscopic oscillations of all the bonds holding matter together. Observed amplitudes of oscillation can be calculated for any temperature using Planck's empirical law. Temperatures in Nature are averaged, not added. Similarly heats, which are also two-dimensional spectra of frequencies and amplitudes, must be averaged not added together. Temperature flows through matter, air, and space only from warm to cool by simultaneous resonance at all frequencies of oscillation. These new insights into temperature, heat, and thermal energy show several fatal problems with greenhouse warming theory and provide new ways to understand sub-microscopic physics.

Keywords: Temperature, heat, thermal energy, warming, radiation, oscillation, kinetic energy, resonance, ozone depletion, greenhouse 
Facing physical reality: Physicists observe, try to understand, and seek to quantify what is physically happening in Nature. Often they must make simplifying assumptions based on scientific tradition, training, and experience. There may be several different ways to visualize and quantify the same observation. In this case, a fundamental problem in physics becomes determining which system of assumptions implemented by which system of mathematics describes most accurately what is actually physically happening in Nature.

For example, for more than 2500 years, natural philosophers and ultimately physicists have debated what is the physical nature of light. Does electromagnetic radiation travel through air and space as waves or as particles? But waves, physically, are defined as temporary deformation of matter and Michelson and Morley $^{2}$ showed definitively in 1887 that there is no matter in space, no luminiferous aether, ${ }^{3}$ through which light can travel as waves.

As for particles, electromagnetic radiation is clearly observed to be a continuum of frequencies of oscillation extending over at least 20 orders of magnitude from radio signals to gamma rays. ${ }^{4}$ How do you physically divide a continuum into myriads of tiny particles? Which decimal place is the ultimate quantum and why? And how, precisely, physically, step by physical step, do these photons interact, for example, with a gas molecule to form well-observed spectral lines of absorption? ${ }^{5}$

During the $20^{\text {th }}$ century, physicists developed the concept of wave-particle duality, the idea that every particle or quantum entity may be described as either a particle or a wave. This concept raises a point of logic: A mule can be described as sort of like a donkey and sort of like a horse, but it is in reality neither - it is a mule. Similarly, light is observed to behave sort of like waves and sort of like particles, but what is light physically?

Furthermore, light is not visible until it interacts with matter. Why do we insist on describing something invisible in terms of things that are visible or at least that we can visualize such as waves or particles?

The closest things to truth in physics are direct observations of what is physically happening in Nature - observations that do not depend on some theory to observe and understand. In this paper, we investigate what can be observed directly regarding electromagnetic radiation, thermal energy, heat, and temperature. This quest for truth leads to a new understanding of what thermal radiation is physically and how absorbing thermal radiation physically heats Earth.

Heat is currently defined as a flux: For more than two centuries, ${ }^{6}$ heat has been thought of as an amount of thermal energy being transferred each second from a warmer body of solid matter to a cooler body of solid matter measured in units of watts per square meter where one watt equals one joule of energy transferred each 
second. ${ }^{7}$ Heat is not considered to be a physical property of either body. Heat is very specifically not considered to be an amount of some undefined substance, although it is widely assumed that the greater the net amount of heat absorbed, the hotter a body will become. Heat is simply defined as a flux, "a concept in applied mathematics and vector calculus, ... a vector quantity, describing the magnitude and direction of the flow of a substance or property."

This definition quantifies a mathematical variable that can be added and subtracted but it avoids addressing the issue of what heat is physically. Heat is an amount of what? In the real world, something is physically being transferred. And if we do not know what is physically being transferred, how can we determine how it is physically being transferred?

The physical effect of the transfer of heat is to decrease the temperature of the emitting hotter body and increase the temperature of the absorbing cooler body. Thus, at the macroscopic level, what is physically being transferred is temperature. But temperature is not additive as flux is assumed to be. If you take two bodies of matter that are identical in every way except for temperature and connect them together, the resulting temperature is the average of the two initial temperatures, not the sum. This implies that the physical processes resulting in temperature are also not additive.

Temperature is averative: All curves of warming or cooling of solid matter are observed to be asymptotic to the final temperature as shown in Figure 1 for warming. The black curve plots the warming measured when shining a light on a small piece of thin black metal. The red line shows the warming calculated by multiplying a constant times the final temperature minus the current temperature. The greater the difference in temperature, the greater the flux of heat, and the faster the body is warmed. The greatest temperature difference is in the beginning. As the temperature difference approaches zero asymptotically over time, it takes a very long time to completely warm the body.

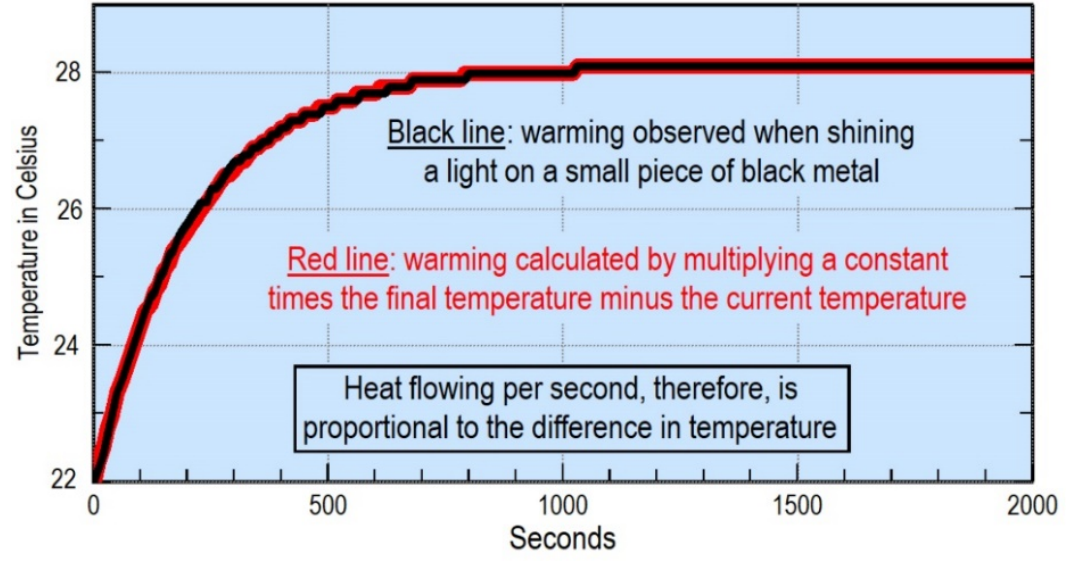

Figure 1. Heat flowing per second is proportional to the difference in temperature at that moment. 
You get this same asymptotic shape when approaching a wall by moving the average distance between you and the wall during each unit of time. You get very close to the wall soon, but you will never theoretically reach the wall, although the difference in distance gradually becomes insignificant.

At each moment in time, the temperature of the absorbing body is averaged with the ultimate temperature, which is determined by the temperature of the emitting body. This averaging is done in Nature by resonance, which will be described below. In this way, temperatures are averative, a word I am coining to emphasize the fundamental distinction in physical reality between adding and averaging when dealing with temperatures, heat, or thermal energy. The resulting temperature is either the average of the two initial temperatures, or, in the most general case, is somewhere between the two initial temperatures depending on the physical properties of both bodies of solid matter and the boundary conditions.

Averaging instead of adding occurs because temperature is an intensive physical property, which means temperature does not depend on the size or amount of matter involved. ${ }^{9}$ Temperature is the result of things happening at the submicroscopic level that are spread evenly throughout the body of matter. Therefore, you can divide a body of matter up into as many pieces as you want and each piece will initially have the same temperature. There is no such thing as a physical amount of temperature. Temperature is simply a level of thermal energy that determines how hot the body is. Since heat is temperature in transit, it makes no physical sense to think of heat macroscopically as a physical amount of anything.

Thermal energy is kinetic energy, which is averative: According to Grossman, ${ }^{10}$ "by measuring temperature, we're measuring how fast the atoms in the material are moving. The higher the average velocity, of the atoms, the higher the temperature of the material."

In a gas, atoms and molecules are free to move independently in any direction until they collide with another atom, molecule, or the walls of a container. Then they typically move independently in another direction. Each atom or molecule can be thought of as "possessing" a kinetic energy of linear motion that is quantified as one-half its mass times its velocity of linear motion squared. It makes no physical sense to add these kinetic energies together because each kinetic energy applies only to one discrete, individual atom or molecule. We observe a distribution of kinetic energies that we typically summarize by thinking in terms of a statistical average. We observe that temperature of a gas increases directly proportional to the statistical average of this distribution of kinetic energies. Kinetic energy of linear motion is averative. 
In solid matter, we observe that all bonds holding matter together oscillate back and forth at trillions $\left(10^{12}\right)$ of cycles per second over amplitudes measured in picometers $\left(10^{-12}\right.$ meters). Physicists think of these oscillators as being driven by the electrodynamic forces of repulsion of like electric charges and the electrodynamic forces of attraction of unlike electric charges as approximated by the Morse potential ${ }^{11}$ shown in Figure 2, or by the more detailed Morse/Long range potential. ${ }^{12}$ These frequencies of oscillation are more than ten million times higher than what we can perceive as oscillations. ${ }^{13} \mathrm{We}$ perceive them as temperature. Electrodynamic forces are

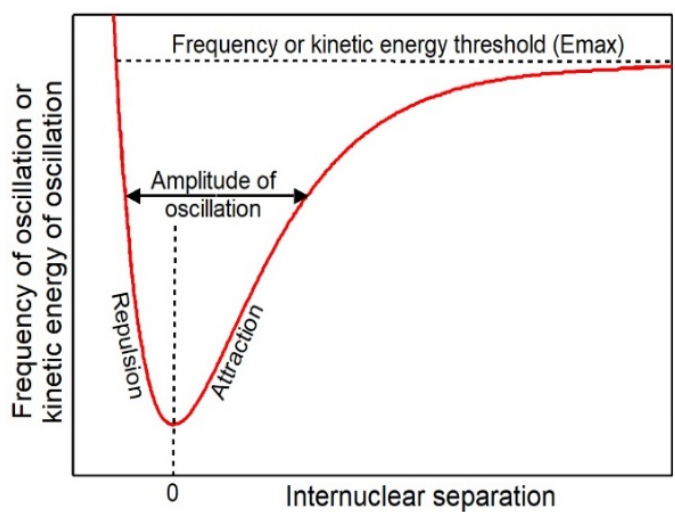

Figure 2. Oscillation of a molecular bond between forces of repulsion and forces of attraction. The molecule is dissociated when the frequency, which is also kinetic energy of oscillation, is equal to Emax.

frictionless, allowing these molecular-bond-scale oscillators to continue to oscillate for exceptionally long periods of time.

Each oscillator is oscillating at some frequency of oscillation with some amplitude of oscillation. We observe that each oscillator on the surface of a piece of solid matter transmits into air and space its frequency of oscillation and its amplitude of oscillation in the same physical manner that a radio transmitter transmits its frequency of oscillation and its amplitude of oscillation by oscillatory motion of charge on the transmitter's antenna. The orientation of the axis of oscillation of each bond will vary, but as with a radio antenna, the resulting electromagnetic field will be greatest perpendicular to the axis of oscillation, as observed.

In this way, thermal radiation, which is defined as radiation emitted by a body of matter because of the body's temperature, is observed to consist of a very broad spectrum of frequencies of oscillation known as the electromagnetic spectrum. ${ }^{4}$ Furthermore, there can be many modes of oscillation for each oscillator and each mode can have numerous overtones or harmonics of oscillation, each with a unique frequency of oscillation. Thermal radiation from even a very small piece of solid matter is the result of simultaneous oscillation of trillions of bonds holding solid matter together.

Each mode of oscillation of each frictionless molecular-bond-scale oscillator can be thought of as "possessing" a kinetic energy of oscillation (E), which Planck ${ }^{14}$ postulated in 1900 is equal to the frequency of oscillation ( $v$, the Greek letter nu) times a constant of proportionality (h), now known as the Planck constant. This constant is simply the kinetic energy of oscillation contained within a frequency of one cycle per second. The Planck constant, the slope of a line through the origin of 
a plot of kinetic energy as a function of frequency, can be estimated easily in a high-school physics laboratory using several different light-emitting diodes oscillating at different frequencies. ${ }^{15}$ What $\mathrm{E}=\mathrm{h} v$ tells us is that frequency of oscillation is physically the same thing as kinetic energy of oscillation times a scaling constant and that kinetic energy of oscillation is physically the same thing as frequency of oscillation times a scaling constant as calculated for all frequencies in the electromagnetic spectrum. ${ }^{4}$ The higher the frequency of oscillation, the higher the velocity of motion of the atoms, and the higher the kinetic energy of oscillation.

It makes no physical sense to add these kinetic energies of oscillation together because each kinetic energy applies only to one discrete, individual, molecularbond-scale oscillator. We observe a distribution of kinetic energies that we can summarize by calculating a statistical average. We observe that temperature of solid matter increases directly proportional to the statistical average of this distribution of kinetic energies of oscillation. Kinetic energy of oscillation is averative.

\section{Planck's empirical law calculates the observed physical properties of temperature: In the late $19^{\text {th }}$ century, many physicists used a glass prism to} spatially separate visible light from various sources into a spectrum of colors. They then moved various sensors through each band of color, carefully measuring changes as a function of temperature of the source of the radiation. For infrared radiation, which does not have enough energy to penetrate glass, they utilized prisms made of halite.

In 1900, Planck ${ }^{14,16}$ was able to devise an equation that calculates accurately the observed measurements at each frequency of oscillation as a function of the temperature of the emitting body, plotted in Figure 3 for Earth at $15^{\circ} \mathrm{C}$, the filament of an incandescent light bulb at $3300^{\circ} \mathrm{C}$, and Sun at $5500^{\circ} \mathrm{C}$. This equation, now known as Planck's empirical law, was determined by trial and error to explain extensive observations. It was not based on theory.

In the 1890 s and still today, physicists thought their sensors were measuring energy of radiation flowing per second, so Planck plotted flux of energy in watts per square meter on the y-axis. What is fascinating, however, is that Planck, in order to satisfy dimensional analysis for his equation, postulated that energy of radiation $(\mathrm{E})$ is equal to the Planck constant (h) times frequency $(v), \mathrm{E}=\mathrm{h} v$, something he called "energy elements." In 1905, Einstein ${ }^{17}$ called them "energy quanta", and in 1926, Lewis ${ }^{18}$ called them "photons". But if energy (E) equals a constant (h) times frequency $(v)$, then energy must be plotted parallel to frequency on an alternative $\mathrm{x}$-axis as shown at the top of Figure 3. 


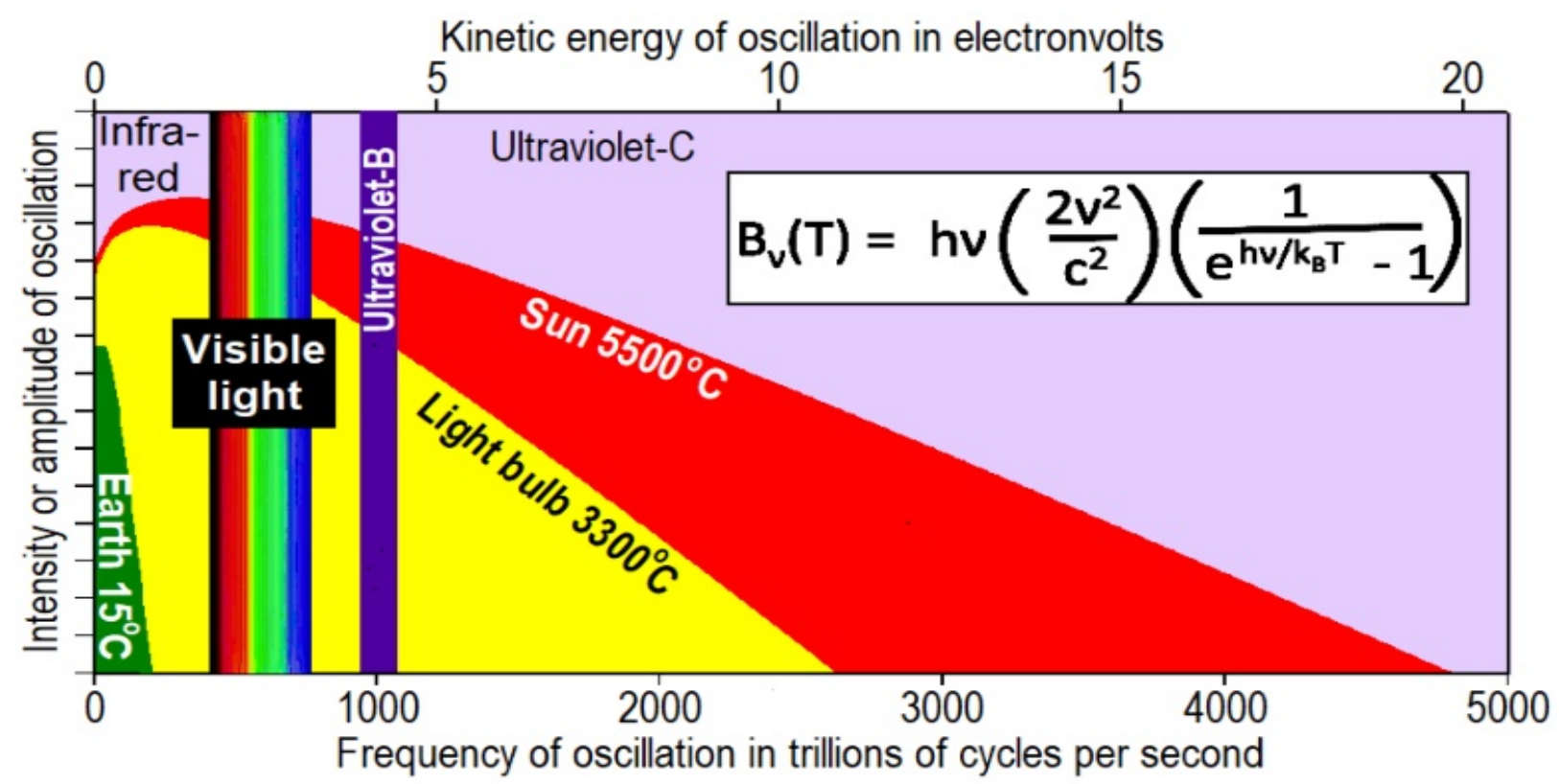

Figure 3. Planck's empirical law calculating the intensity or amplitude of oscillation at each frequency of oscillation as a function of the temperature of the radiating body.

In 1900, Planck considered $\mathrm{E}=\mathrm{h} v$ to be a "mathematical trick or convenience" and his empirical law to be a "fortunate guess". ${ }^{19} \mathrm{He}$ never wondered in writing about what energy of radiation is physically. Nevertheless, in 1918, Planck earned the Nobel Prize in Physics for "his discovery of energy quanta." ${ }^{20}$ In 1931, he admitted that introducing the "energy element" in 1900 was "a purely formal assumption and I really did not give it much thought except that no matter what the cost, I must bring about a positive result". ${ }^{21}$ In other words, Planck, a theoretical physicist, was obsessed with the mathematics rather than thinking carefully about what was actually physically happening in Nature. He did think mathematically in terms of tiny oscillators, which he called resonators, but he was confused by the widespread assumptions that energy should be plotted on the y-axis and wavelength on the $\mathrm{x}$ axis. Wavelength and wave frequency assume the wave theory of light. What we measure is frequency of oscillation, which has nothing to do with waves.

There are two ways to look at the equation $\mathrm{E}=\mathrm{h} v$. First as a level of energy contained in a single frequency - the minimum level of energy (Emax in Figure 2) required to break the bond of an electron in the photoelectric effect or to break a bond of a molecule causing dissociation. For example, a molecule of oxygen is observed to be dissociated into two atoms of oxygen when it absorbs a frequency close to 1237 terahertz ( $10^{12}$ cycles per second). In this case, Emax can be thought of as the minimum level of energy required to cause a photochemical reaction, something Einstein called a "light quantum". ${ }^{17}$ 
The second way to look at the equation $\mathrm{E}=\mathrm{h} v$ is to recognize that frequency $(v)$ is a very broad spectrum of frequencies - the electromagnetic spectrum. ${ }^{4} \mathrm{~A}$ constant times a spectrum must equal a spectrum. Therefore, energy $(\mathrm{E})$ is a broad spectrum of energies, the opposite of a "light quantum" or "photon."

Frictionless oscillators have two primary physical properties: frequency of oscillation and amplitude of oscillation. Thus, at the sub-microscopic level, Planck's empirical law calculates amplitude of oscillation on the y-axis. At the macroscopic level, our eyes perceive amplitude of oscillation as intensity or brightness. Intensity of radiant energy is currently defined as power transferred per unit area in units of watts per square meter, a flux, so that needs to be changed. Brightness is defined as an attribute of visual perception. We will see below that amplitude of oscillation is what makes things we see look brighter. I simply plot orders of magnitude on the y-axis in Figure 3 because I think it would be best for the absolute values of amplitude of oscillation measured in picometers to be calibrated in the laboratory. They could vary in detail with chemical composition.

\section{Heat physically is what a body of solid matter must absorb to become}

warmer: Planck's empirical law not only shows the physical properties of thermal radiation and the physical properties of oscillators on the surface of solid matter, but also the physical properties that must exist throughout a body of solid matter at thermal equilibrium for that body to "possess" a temperature. Note from Figure 3 that the higher the temperature of the radiating body, the higher the observed amplitude of oscillation at each and every frequency of oscillation and the higher the observed frequencies with the greatest amplitudes of oscillation.

Heat can be defined most fundamentally as that which a body of solid matter must absorb to get warmer and must emit to get cooler. In Figure 3, Earth (blue) must absorb the physical properties shaded yellow to become as hot as the filament of an incandescent light bulb. Heat, physically, is thus a two-dimensional spectrum of values quantified by subtracting Planck's law for the cooler body from Planck's law for the warmer body. This explains why a body can only be warmed by absorbing radiation if that radiation comes from a warmer body, and why the rate that heat flows is determined by the difference in temperature.

Amplitude of oscillation flows by resonance, a fundamental physical property of oscillatory systems: Temperature in solid matter, as described by Planck's empirical law (Figure 3), is the result of oscillation of all the bonds holding a piece of solid matter together. The most important physical property of oscillating systems beyond frequency of oscillation and amplitude of oscillation is resonance, also known as sympathetic oscillation. When two discrete molecular-bond-scale oscillators are oscillating at the same frequency and are within line-of-sight of each 
other or mechanically connected in some way, they are observed to share amplitude of oscillation. The oscillator with the largest amplitude of oscillation loses amplitude of oscillation to the oscillator with the least amplitude of oscillation. In the simplest case, both oscillators end up with the average of the initial amplitudes of oscillation. But in undamped systems with no friction, such as molecular-bond-scale oscillators, the increase in amplitude can be

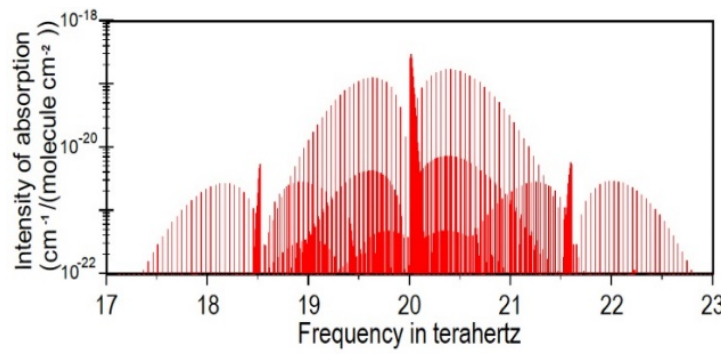

Figure 4. Spectral lines of absorption by a molecule of carbon dioxide in the frequency range of 17 to 23 terahertz, commonly referred to as centered around 14.9 micrometers wavelength. larger if that specific frequency is a natural resonant frequency of that specific oscillator, explaining well-documented spectral lines of absorption for gas molecules (Figure 4). ${ }^{5}$

When this sharing of amplitude is done simultaneously across all frequencies of oscillation, the temperature of the hotter body becomes a little cooler and the temperature of the cooler body becomes a little warmer. What is "flowing" is amplitude of oscillation at each and every frequency of oscillation. Thus, for solid matter, when we measure temperature, we are measuring amplitude of oscillation at each and every frequency of oscillation as calculated by Planck's empirical law (Figure 3). The higher the temperature, the higher the dominant frequencies of oscillation, the higher the amplitude of oscillation at each and every frequency of oscillation, and the higher the average velocity of oscillatory motion.

All frequencies throughout the electromagnetic spectrum coexist at all times and at all locations. What we observe varies in time and space for visible light is the intensity of oscillation caused by the amplitude of oscillation at each frequency of oscillation ranging on a logarithmic scale from completely insignificant to dominant.

Because all these sub-microscopic oscillators are frictionless, the only known physical way to increase or decrease the amplitude of oscillation of any molecularbond-scale oscillator is via resonance. Therefore, the only way for heat to physically flow is by resonance.

Resonance is all around us. We see by resonance. Each of our eyes contains six to seven million cone cells ${ }^{22}$ in sets of three that resonate to visible light in slightly different ways shown by the solid lines in Figure 5 labelled Red, Green, and Blue. Our brain processes the three slightly different neurological signals to detect ten million different shades of color. All cone cells resonate simultaneously, each with 
a single oscillating molecular bond within your field of view, allowing you to see a whole scene in full color.

We hear by resonance when the tiny hair cells ${ }^{23}$ in our inner ear resonate at different frequencies of air pressure, transmitting that precise frequency to our brains. Scientists are beginning to realize, similarly, that a small number of sensor types involving resonance may be what enables animals to recognize a very wide range of smells and tastes. ${ }^{24-27}$ We feel temperature by resonance. Resonance appears to be the primary way living things interact with their physical and social environments.

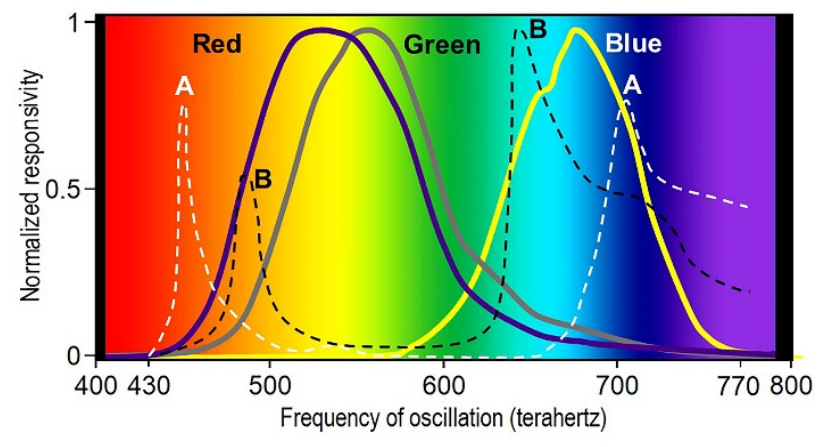

Figure 5. Sets of three cones in your eyes respond in slightly different ways to Red, Green, and Blue light as shown by the solid curves. A leaf appears green because chlorophyll $A$ and $B$ absorb most red and blue light as shown by the dashed lines, leaving only oscillations in the frequency range of green on the surface to resonate with the cells in your eyes. $^{l}$

Frequency of oscillation, which is the same physical thing as energy of radiation, is well observed not to change with distance, even galactic distances, except for Doppler effects. Amplitude of oscillation or intensity of radiation, on the other hand, is well observed to decrease with the square of the distance travelled. This decrease can be understood in terms of the apparent density of molecular-bondscale oscillators on the surface of near and distant bodies. Over short distances, there is a one-to-one correspondence between oscillators. As distance increases, the distant object looks smaller and smaller. Fewer and fewer molecules on the distant surface are able to resonate with each molecule on the near surface. Thus, the amplitude transferred by resonance must then be shared by conduction with more and more similar oscillators on the distant surface as they reach thermal equilibrium. In this way, the rate of amplitude transfer decreases with the square of increasing distance.

Resonance provides a physical explanation for how, physically, a warming system "knows" the final temperature as plotted in Figure 1, how, physically, amplitudes of oscillation and temperatures are averaged, and how, physically, Planck curves keep their shape. Resonance explains why heat can only flow from hot to cold. Resonance shows that a body of matter does not emit the same radiation in every direction as commonly assumed. Resonance is point to point. For example, Earth, on sides looking at Sun, absorbs heat, amplitude of oscillation, from a hotter Sun, but on sides not looking at Sun, Earth loses a lot of heat at the same time to much colder deep space. We have a lot to learn about the physics of resonance including 
how it physically happens over all distances, how rapidly amplitudes are changed, and the effects of the physical properties of the materials involved.

Ozone depletion explains observed warming in considerable detail: The world warmed $0.6^{\circ} \mathrm{C}$ from 1970 to 1998 and $0.3^{\circ} \mathrm{C}$, five times faster, from 2014 to $2016 .^{28}$ There was no significant warming from 1950 to 1970, from 1998 to 2013, and since 2016, more than half the time since 1950, even though concentrations of carbon dioxide kept rising at ever increasing rates. ${ }^{29}$ There is no direct correlation between observed sudden changes in rates of warming around 1970, 1998, 2014, and 2016 and changes in emission of carbon dioxide. More than 50 peer-reviewed papers seek to explain the global warming hiatus ${ }^{30,31}$ from 1998 through 2013 in terms of greenhouse gases without much agreement. ${ }^{32}$

These changes in warming, however, and most periods of sudden global warming throughout Earth history, can be explained in detail by depletion of the ozone layer. ${ }^{33-35}$ When the ozone layer is depleted, less ultraviolet-B radiation than usual is absorbed by ozone within the ozone layer, cooling the ozone layer as observed. ${ }^{33,36}$ More ultraviolet-B is then observed to reach Earth's surface. Ultraviolet-B is the highest frequency, most energetic, "hottest" solar radiation reaching Earth (Figure 3) where it is observed to cause sunburn, skin cancer, cataracts, mutations, and dissociation of ground-level ozone pollution leading to warmer temperatures. This dissociation of pollution explains the urban heat island effect $^{37}$ and why warming since 1970 was twice as great ${ }^{28}$ in the northern hemisphere containing $90 \%$ of global population. Ultraviolet-B penetrates oceans tens of meters ${ }^{38}$ and is therefore absorbed very efficiently, raising ocean heat content ${ }^{39}$ substantially as observed since ozone depletion began increasing around 1970. The greatest warming observed on Earth is along the Antarctic Peninsula and in southern oceans, both within the Antarctic ozone hole, the region with greatest observed ozone depletion. ${ }^{40}$

In the late 1960s, humans began manufacturing large amounts of chlorofluorocarbon gases (CFCs). In 1974, scientists discovered when CFCs reach the stratosphere, these very inert molecules can be broken down by solar ultraviolet radiation, releasing atoms of chlorine. ${ }^{41}$ One atom of chlorine at very cold temperatures can lead to destruction of 100,000 molecules of ozone, ${ }^{41}$ causing CFCs to be the Achilles heel of climate change.

When the Antarctic ozone hole was discovered in $1985,{ }^{42}$ scientists and political leaders worked effectively together at the United Nations to frame and pass the Montreal Protocol in 1987, mandating cutback in CFC production beginning in 1989. By 1993, concentrations of CFCs in the atmosphere stopped increasing. By 1995, ozone depletion stopped increasing. By 1998, average global temperatures 
stopped increasing. Humans, by manufacturing CFCs, had caused the world to warm $0.6^{\circ} \mathrm{C}$ from 1970 to 1998 . Humans, by passing the Montreal Protocol stopped this increase in warming in 1998, completing the most definitive experiment ever done concerning the effect of a concentration of a gas on global temperatures. It is expected to take many decades for the ozone layer to recover. ${ }^{43}$ Without the Montreal Protocol, average global temperatures today would probably be at least $0.5^{\circ} \mathrm{C}$ warmer. ${ }^{44}$

In 2014, Bárðarbunga volcano in central Iceland extruded the largest basaltic lava flow since 1783. ${ }^{45,46}$ Extensive flows of basaltic lava release ten-times more chlorine $^{47,48}$ than explosive magmas and large amounts of hot air capable of convecting chlorine up into the ozone layer. Global warming increased rapidly $0.3^{\circ} \mathrm{C}$ from 2014 to 2016 , the hottest year on record. Throughout Earth history, extensive extrusion of basaltic lava flows covering areas ranging from tens, to thousands, to millions of square kilometers were contemporaneous with major global warming, the larger the flow, the greater the warming. ${ }^{33-35,49}$ The extensive evidence for sudden warming by ozone depletion throughout Earth history is described in detail at OzoneDepletionTheory.info.

Why greenhouse-warming theory is mistaken: Greenhouse-warming theory assumes that increasing atmospheric concentrations of greenhouse-gases absorbing increasing amounts of infrared radiation from Earth cause global warming in one way or another. But warming of solid matter is not caused by increasing amounts of anything. It is caused by increasing the amplitude of oscillation at each and every frequency of oscillation as shown in Figure 3. No body of matter can be warmed by its own radiation because its own radiation does not contain the increases in amplitude of oscillation at each frequency of oscillation required for heat to flow. If bodies could be warmed by their own radiation, we could have unlimited free thermal energy.

Greenhouse-warming theory is based on the assumptions that heat is a flux, an amount per second measured in units of watts per square meter, and that these fluxes can be added and subtracted. As shown in Figure 3, heat is clearly observed to be a two-dimensional spectrum of frequencies of oscillation and associated amplitudes of oscillation quantified as the difference between the Planck curve for hotter body minus the Planck curve for the cooler body. Thermal energy, heat, and temperature are each observed to be averative. They are not additive. They each cannot be summed. Flux, the rate that heat flows per unit time, is well-observed in Figure 1 to be directly proportional to the difference in temperature, which is proportional to the difference in amplitude of oscillation at each frequency of oscillation (Figure 3). 
Furthermore, greenhouse gases do not absorb heat, which is a broad spectrum of frequencies of oscillation and related amplitudes of oscillation shown in Figure 3. Greenhouse gases are clearly observed to absorb only the resonant frequencies of the bonds holding the molecule together. Absorption of these spectral lines has no direct effect on temperature of a gas. Carbon dioxide, for example, absorbs less than $16 \%$ of the infrared frequencies radiated by Earth. The spectral lines of absorption for most of these resonant frequencies of the molecule are shown in Figure $4 .{ }^{5,50}$ Yet to change the temperature of a body of matter, the body must absorb heat that increases amplitudes of oscillation at each and every frequency of oscillation as shown by Planck's empirical law in Figure 3.

Some scientists assume that this resonant oscillatory energy absorbed into the bonds is converted to linear kinetic energy of the molecule by myriads of collisions according to the law of equipartition. Yet equipartition is not very efficient and any such increase in linear kinetic energy of one molecule of carbon dioxide would then need to be shared with the 2500 other atoms and molecules making up the concentrations found in air. Greenhouse gases absorbing terrestrial infrared radiation have never been shown by experiment to cause warming of air as explained at JustProveCO2.com. Experiments claiming to show warming typically use heat sources such as Sun or light bulbs that are much hotter than infrared radiation radiated by Earth.

Greenhouse-warming theory is based on the assumption that the primary difference between radiation from Sun and Earth is the amount of flux emitted. Planck's empirical law, however, shows in Figure 3 that the physical properties of radiation from Sun plotted in red, yellow, and blue are very different from the physical properties of radiation from Earth plotted in blue.

Greenhouse-warming theory does not take into account the widely-accepted reality stated in the Planck-Einstein relation ${ }^{51}$ that radiant kinetic energy (E) is equal to the Planck constant (h) times frequency of oscillation $(v) . \mathrm{E}=\mathrm{h} v$ says that any amount of ultraviolet-B radiation has fifty times the kinetic energy of any amount of infrared radiation absorbed most strongly by carbon dioxide. For this reason, greenhouse warming theory grossly overestimates the thermal effect of infrared radiation absorbed by greenhouse gases.

There are only two ways in Earth's atmosphere that air is observed to be heated tens of degrees every day. Air in the troposphere is warmed daily when it comes in contact with Earth's sun-warmed surface and rises by convection, heating the troposphere from below. Air in the stratosphere, however, is observed to be heated from above primarily by ultraviolet-C solar radiation causing dissociation of oxygen, carbon dioxide, and other molecules and ultraviolet-B radiation causing 
dissociation of ozone. Upon dissociation, the pieces of the molecule fly apart at high velocity, increasing the average linear kinetic energy of air, which increases the temperature. Air temperature in the stratosphere increases from around $-56^{\circ} \mathrm{C}$ at the tropopause, 9 to $17 \mathrm{~km}$ above Earth, to around $-15^{\circ} \mathrm{C}$ at the stratopause, 50 to $55 \mathrm{~km}$ above Earth. In this way, dissociation is observed to convert kinetic energy of oscillation of a molecular bond directly, completely, and efficiently into kinetic energy of linear motion, increasing the temperature of a gas. Infrared radiation from Earth does not possess a high-enough level of energy to cause dissociation of any gas molecules.

Many climate scientists argue that Earth's average temperature would be $-19^{\circ} \mathrm{C}$ were it not for a blanket of greenhouse gases raising it to $14^{\circ} \mathrm{C} .{ }^{52} \mathrm{~A}$ blanket slows cooling but cannot cause warming. This warming is clearly observed to occur in the stratosphere primarily by dissociation of molecular oxygen making up $21 \%$ of the atmosphere. Similarly, the atmosphere of Venus is most likely warmed to $463^{\circ} \mathrm{C}$ by dissociation of carbon dioxide making up $96 \%$ of that atmosphere.

In conclusion, there is no climate crisis: Warming of $0.9^{\circ} \mathrm{C}$ since 1950 has caused problems that we have been able to adapt to. Numerous climate models, based on greenhouse-warming theory, however, predict three more degrees of warming by the end of this century (Figure 6). ${ }^{53}$ It is this predicted warming that worries so many climate scientists and creates the urgency to act now to reduce greenhouse-gas emissions. But greenhouse-warming theory is not only mistaken, it is not even physically possible as explained in this paper and at Physically-Impossible.com. The warming predicted by these models cannot and will not happen. Ozone-depletion theory, on the other hand, predicts slow cooling over the rest of this century as the ozone layer slowly recovers to pre-1970 levels. ${ }^{43} \mathrm{We}$ may burn fossil fuels safely provided we minimize pollution.

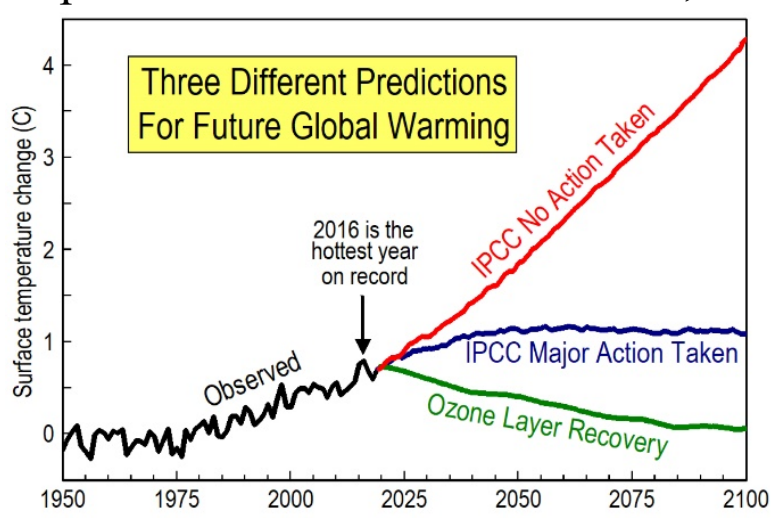

Figure 6. The Intergovernmental Panel on Climate Change (IPCC) predicts several degrees of warming by 2100 (red line). They predict that if major action is taken immediately to reduce greenhouse-gas emissions, warming might be kept to only one an additional degree (blue line). Ozone depletion theory, on the other hand predicts gradual cooling of climate over the next several decades unless there is a major new source of ozone depletion (green line). 
Acknowledgments: This work was funded by my children's inheritance.

Declaration of Interests: The author declares no competing interests.

\section{References:}

1. Wikipedia (2020). Chlorophyll, https://en.wikipedia.org/wiki/Chlorophyll.

2. Michelson, A.A., and Morley, E.W. (1887). On the relative motion of the earth and the luminiferous ether. Am J Sci 34, 333-345. https://dx.doi.org/10.2475/ajs.s3-34.203.333

3. Wikipedia (2020). Luminiferous aether, https://en.wikipedia.org/wiki/Luminiferous aether.

4. Wikipedia (2020). Electromagnetic spectrum, https://en.wikipedia.org/wiki/Electromagnetic spectrum.

5. Gordon, I.E., Rothman, L.S., Hill, C., Kochanov, R.V., Tan, Y., Bernath, P.F., Birk, M., Boudon, V., Campargue, A., and Chance, K. (2017). The HITRAN2016 molecular spectroscopic database. J Quant Spectrosc Radiat Transfer 203, 3-69.

https://doi.org/10.1016/j.jqsrt.2017.06.038.

6. Thompson, B. (1798). An experimental enquiry concerning the source of the heat which is excited by friction. Philosphical Transactions of the Royal Society 88, 80-102.

https://doi.org/10.1098/rstl.1798.0006.

7. Wikipedia (2020). Heat, https://en.wikipedia.org/wiki/Heat.

8. Wikipedia (2020). Flux, https://en.wikipedia.org/wiki/Flux.

9. Wikipedia (2020). Intensive and extensive properties, https://en.wikipedia.org/wiki/Intensive and extensive properties.

10. Grossman, J.C. (2014). Thermodynamics: Four laws that move the Universe, https://www.thegreatcourses.com/courses/thermodynamics-four-laws-that-move-theuniverse.html.

11. Morse, P.M. (1929). Diatomic molecules according to the wave mechanics. II. Vibrational levels. Phys Rev 34, 57-64. https://dx.doi.org/10.1103/PhysRev.34.57.

12. Le Roy, R.J., Dattani, N.S., Coxon, J.A., Ross, A.J., Crozet, P., and Linton, C. (2009). Accurate analytic potentials for $\mathrm{Li}_{2}\left(\mathrm{X}^{1} \Sigma+\mathrm{g}\right)$ and $\mathrm{Li}_{2}\left(\mathrm{~A}^{1} \Sigma^{+\mathrm{u}}\right)$ from 2 to $90 \AA$, and the radiative lifetime of Li(2 p). J Chem Phys 131, 204309. https://dx.doi.org/10.1063/1.3264688.

13. Guignard, J. (1971). Human sensitivity to vibration. Journal of sound and vibration $15,11-$ 16. https://doi.org/10.1016/0022-460X(71)90354-3.

14. Planck, M. (1900). On the Theory of the Energy Distribution Law of the Normal Spectrum. Verhandlungen der Deutschen Physikalischen Gesellschaft 2.

http://hermes.ffn.ub.es/luisnavarro/nuevo maletin/Planck\%20(1900),\%20Distribution\%20 Law.pdf.

15. Rute, M., and Sérgio, P. (2014). Classroom fundamentals: measuring the Planck constant. Science in School, 28-33.

https://SciencelnSchool.org/sites/default/files/teaserPdf/issue28 planck.pdf.

16. Planck, M. (1900). Entropie und Temperatur strahlender Wärme. Ann Phys 306, 719-737. https://dx.doi.org/10.1002/andp.19003060410.

17. Einstein, A. (1905). On a heuristic point of view about the creation and conversion of light. Ann Phys 322, 132-148. 
https://en.wikisource.org/wiki/Translation:On a Heuristic Point of View about the Cre ation and Conversion of Light.

18. Lewis, G.N. (1926). The conservation of photons. Nature 118, 874-875. https://dx.doi.org/10.1038/118874a0.

19. Gearhart, C.A. (2008). Max Planck and black-body radiation. In Max Planck: Annalen Papers, D. Hoffmann, ed. (Wiley-VCH), pp. 395-418.

20. Nobel Prize in Physics (1918). https://www.nobelprize.org/prizes/physics/1918/summary/.

21. Kragh, H. (2000). Max Planck: the reluctant revolutionary. PhysicsWorld 13(12); . https://dx.doi.org/10.1088/2058-7058/13/12/34.

22. Wikipedia (2020). Cone cell, https://en.wikipedia.org/wiki/Cone cell.

23. Wikipedia (2020). Hair cell, https://en.wikipedia.org/wiki/Hair cell.

24. Burr, C. (2004). The emperor of scent: A true story of perfume and obsession (Random House).

25. Piesse, G.W.S. (2015). The art of perfumery: Method of obtaining the odors of plants (CreateSpace Independent Publishing Platform).

26. Bushdid, C., Magnasco, M.O., Vosshall, L.B., and Keller, A. (2014). Humans can discriminate more than 1 trillion olfactory stimuli. Science 343, 1370-1372. https://doi.org/10.1126/science.1249168.

27. Carney, R.S. (2019). Parallel and Sequential Sequences of Taste Detection and Discrimination in Humans. eNeuro 6(1); · https://doi.org/10.1523/ENEURO.0010-19.2019.

28. NOAA (2020). Global temperature data sets: Overview \& comparison table, https://climatedataguide.ucar.edu/climate-data/global-temperature-data-sets-overviewcomparison-table.

29. NOAA (2020). Annual mean $\mathrm{CO}_{2}$ at Mauna Loa. https://www.esrl.noaa.gov/gmd/ccgg/trends/\#mlo full.

30. Wikipedia (2020). Global warming hiatus, https://en.wikipedia.org/wiki/Global warming hiatus.

31. Stocker, T.F., Qin, D., Plattner, G.-K., Alexander, L.V., Allen, S.K., Bindoff, N.L., Bréon, F.-M., Church, J.A., Cubasch, U., Emori, S., et al. (2013). Technical Summary. In Climate Change 2013: The Physical Science Basis Contribution of Working Group I to the Fifth Assessment Report of the Intergovernmental Panel on Climate T.F. Stocker, and G.-K.P. D. Qin, M. Tignor, S.K. Allen, J. Boschung, A. Nauels, Y. Xia, V. Bex and P.M. Midgley, eds. (Cambridge, United Kingdom and New York, NY, USA: Cambridge University Press), pp. 33-115. http://www.climatechange2013.org/spm.

32. Ward (2020). Published papers discussing the global warming hiatus, https://ozonedepletiontheory.info/gg-warming-hiatus.html.

33. Ward, P.L. (2016). Ozone depletion explains global warming. Current Physical Chemistry 6, 275-296. https://dx.doi.org/10.2174/1877946806999160629080145.

34. Ward, P.L. (2016). What really causes global warming? Greenhouse gases or ozone depletion? (New York: Morgan James Publishing).

35. Ward, P.L. (2020). What really causes global warming? Greenhouse gases or ozone depletion?, https://whyclimatechanges.com/.

36. Ward, P.L. (2020). Ozone concentrations observed since 1927, https://ozonedepletiontheory.info/ozone-depletion.html. 
37. Wikipedia (2020). Urban heat island, https://en.wikipedia.org/wiki/Urban heat island.

38. Tedetti, M., and Sempéré, R. (2006). Penetration of ultraviolet radiation in the marine environment. A review. Photochem Photobiol 82, 389-397.

http://dx.doi.org/10.1562/2005-11-09-IR-733.

39. Levitus, S., Antonov, J., Boyer, T., Baranova, O., Garcia, H., Locarnini, R., Mishonov, A., Reagan, J., Seidov, D., and Yarosh, E. (2012). World ocean heat content and thermosteric sea level change (0-2000 m), 1955-2010. Geophys Res Lett 39, L10603.

https://dx.doi.org/10.1029/2012GL051106.

40. Ward, P.L. (2020). The greatest warming occurred where and when ozone was most depleted, https://ozonedepletiontheory.info/greatest-warming-observed.html.

41. Molina, M.J., and Rowland, F.S. (1974). Stratospheric sink for chlorofluoromethanes:

Chlorine catalysed destruction of ozone. Nature 249, 810-814.

https://dx.doi.org/10.1038/249810a0.

42. Farman, J.C., Gardiner, B.G., and Shanklin, J.D. (1985). Large losses of total $\mathrm{O}_{3}$ in atmosphere reveal seasonal $\mathrm{ClO}_{x} / \mathrm{NO}_{x}$ interaction. Nature 315, 207-210.

https://dx.doi.org/10.1038/315207a0.

43. Solomon, S. (1999). Stratospheric ozone depletion: A review of concepts and history. Rev Geophys 37, 275-316. https://dx.doi.org/10.1029/1999RG900008.

44. Ward, P.L. (2016). J8.3 Mario Molina's Research Not Only Led to the Solution for the Antarctic Ozone Hole, But it Led to the Solution for Global Warming Caused by Humans Since 1960. In American Meteorological Society Annual Meeting, Abstract:

https://amsconfexcom/ams/96Annual/webprogram/Paper284695html, Video: https://youtube/NF438LDeqLA.

45. Gudmundsson, M.T., Jónsdóttir, K., Hooper, A., Holohan, E.P., Halldórsson, S.A., Ófeigsson, B.G., Cesca, S., Vogfjörd, K.S., Sigmundsson, F., Högnadóttir, T., et al. (2016). Gradual caldera collapse at Bárdarbunga volcano, Iceland, regulated by lateral magma outflow. Science 353, 262. http://dx.doi.org/10.1126/science.aaf8988.

46. Thordarson, T., and Self, S. (2003). Atmospheric and environmental effects of the 17831784 Laki eruption: A review and reassessment. J Geophys Res 108, 4011. http://dx.doi.org/10.1029/2001jd002042.

47. Jambon, A., Deruelle, B., Dreibus, G., and Pineau, F. (1995). Chlorine and bromine abundance in MORB: the contrasting behaviour of the Mid-Atlantic Ridge and East Pacific Rise and implications for chlorine geodynamic cycle. Chem Geol 126, 101-117. https://doi.org/10.1016/0009-2541(95)00112-4.

48. Self, S., Blake, S., Sharma, K., Widdowson, M., and Sephton, S. (2008). Sulfur and chlorine in late Cretaceous Deccan magmas and eruptive gas release. Science 319, 1654-1657. http://dx.doi.org/10.1126/science.1152830.

49. Ward, P.L. (2020). The ozone depletion theory of global warming, https://ozonedepletiontheory.info/.

50. Ångström, K. (1900). Ueber die Bedeutung des Wasserdampfes und der Kohlensäure bei der Absorption der Erdatmosphäre. Ann Phys 308, 720-732.

https://dx.doi.org/10.1002/andp.19003081208. In English at https://ozonedepletiontheory.info/Papers/Angstrom1900-English.pdf. 
51. Wikipedia (2020). Planck-Einstein relation, https://en.wikipedia.org/wiki/Planck\%E2\%80\%93Einstein relation.

52. Le Treut, H., Somerville, R., Cubasch, U., Ding, Y., Mauritzen, C., Mokssit, A., Peterson, T., and Prather, M. (2007). Historical Overview of Climate Change. In Climate Change 2007: The Physical Science Basis Contribution of Working Group I to the Fourth Assessment Report of the Intergovernmental Panel on Climate Change, S. Solomon, D. Qin, M. Manning, Z. Chen, M. Marquis, K.B. Averyt, M. Tignor, and H.L. Miller, eds. (Cambridge University Press), p. 97. https://www.ipcc.ch/site/assets/uploads/2018/03/ar4-wg1chapter1.pdf.

53. Collins, M., Knutti, R., Arblaster, J., Dufresne, J.-L., Fichefet, T., Friedlingstein, P., Gao, X., Gutowsk, W.J., Johns, T., Krinner, G., et al. (2013). 12. Long-term Climate Change:

Projections, Commitments and Irreversibility. In Climate Change 2013: The Physical Science Basis Contribution of Working Group I to the Fifth Assessment Report of the Intergovernmental Panel on Climate Change, T.F. Stocker, D. Qin, G.-K. Plattner, M. Tignor, S.K. Allen, J. Boschung, A. Nauels, Y. Xia, V. Bex, and P.M. Midgley, eds. (Cambridge, United Kingdom and New York, NY: Cambridge University Press), pp. 1029-1136.

https://doi.org/10.1017/CBO9781107415324.024. 